\title{
Article \\ Spectral and Spatial Cloud Detection Onboard for Hyperspectral Remote Sensing Image
}

\author{
Haoyang $\mathrm{Li}^{1}{ }^{1 *}$, Hong Zheng ${ }^{1}$, Chuanzhao Han ${ }^{2}$, Haibo Wang ${ }^{3}$ and Miu Min ${ }^{4}$ \\ 1 School of Automation Science and Electrical Engineering, Beihang University, No.37 Xueyuan Road, Beijing \\ 100191, China; lhyang@buaa.edu.cn (H.L.); julyanna@vip.sina.com (H.Z.) \\ 2 China Academy of Space Technology, CASA, No.104 Youyi Road, Beijing 100094, China; \\ hanchuanzhao_casc@foxmail.com(C.H.) \\ 3 China Centre for Resources Satellite Data and Application, No.5 Fengxian East Road, Beijing 100094, China; \\ bo3861926@sina.com \\ 4 School of Mathmatics and System Science, Beihang University, No.37 Xueyuan Road, Beijing 100191, China; \\ miaomin@buaa.edu.cn (M.M.) \\ * Correspondence: lhyang@buaa.edu.cn; Tel.: +86-10-8231-6970
}

\begin{abstract}
It is strongly desirable to accurately detect the clouds in hyperspectral images onboard before compression. However, conventional onboard cloud detection methods are not appropriate to all situation such as shadowed cloud or darken snow covered surfaces which are not identified properly in the NDSI test. In this paper, we propose a new spectral-spatial classification strategy to enhance the orbiting cloud screen performances obtained on hyperspectral images by integrating threshold assisted exponential spectral angle map (TESAM), adaptive Markov random fields (aMRFs) and dynamic stochastic resonance (DSR). First, TESAM is performed to classify the cloud pixels coarsely based on spectral information. Then aMRFs is performed to do optimal process by using spatial information, which improved the classification performance significantly. But some misclassification points still exist after aMRFs processing because of the noise of data in the onboard environment. DSR is used to eliminate misclassification points in binary labeling image after aMRFs. Taking level 0.5 data from hyperion as dataset, the average overall accuracy of the proposed algorithm is $96.28 \%$ after test. The method can provide an accurate cloud mask for the on-going EO- 1 images and the similar satellites with the same spectral settings without manual intervention. The experiment indicate that the proposed method reveals better performance than the classical onboard cloud detection or current advanced hyperspectral classification methods.
\end{abstract}

Keywords: onboard cloud detecion; region of interesting compression; themodynamic phase; spectral angle map; markov random field; dynamic stochastic resonance

\section{Introduction}

Future earth science missions will face unprecedented data volumes. Data product sizes and production rates have steadily increased due to improvements in detector, optics, and onboard data handling technology. With the development of Hyperspectral remote sensing technology, hyperspectral imaging techniques have been widely used in many applications, including astronomy, agriculture, biomedical imaging, mineralogy, surveillance, etc. It is used in the spacecraft or aircrafts to get characteristics of target element through capturing image in a large number of narrow and contiguous bands. Typically, one hyperspectral image dataset has over 200 spectral bands, which presents a challenge for both data transmission and storage. [1]. Given the fact that almost all these sensors have only limited memory capacity, data transmission from the sensors to the ground become inevitable for the further data analysis[2]. The large data volumes affect mission requirements for the entire data handling chain, including onboard digitization, storage, downlink, ground processing, and distribution[3]. Bottlenecks along this path can constrain the instrument duty cycle, reducing science and application yield[4]. 
Data compression techniques offer a good solution to the problem. While lossy compression methods typically provide much larger data reduction than lossless methods, they might not be suitable for hyperspectral images used in many accuracy demanding applications, where the images are intended to be analyzed automatically by computers[5]. In particular, bandwidth constraints have motivated new advanced lossless compression techniques such as the FL algorithm[6][7][8] that have achieved compression rates of four or greater. Efforts to optimize lossless methods eventually face theoretical limits, but data rates continue to increase. The challenge has driven research into other techniques that can further reduce data volumes while preserving science yield.

For a specific application, it is very likely that only some regions of the entire image carry the information of interest. Rather than compressing the entire image, sometimes we just need to compress only ROIs in the image. Recently, a new concept known as "remote sensing image with no-data regions compression" has been proposed for the first time[9]. The no-data regions do not provide useful information to some applications, compression methods will benefit significantly from simply not compressing those no-data regions for the sake of a higher compression ratio.

Most remotely sensed images encompass the presence of clouds that, especially in the visible and infrared range, strongly affect the received electromagnetic radiation. Historically, clouds are estimated to cover $54 \%$ or more of the Earth's land area and $68 \%$ or more of the oceans[10][11][12]. Depending on the application, clouds act as an unwanted corruption[13][14] or, as an information source for weather forecast[15]. These cloud-region data were regarded as the arbitrary shape no-data region, which can be compressed[16]. In the ROI map, binary attributes represent cloud and ground respectively pixel by pixel and binary map will be generated to assist in the actual compression. And each binary ROI map can be compressed using ARLE[17]. Since the ROI map can be compressed to a fairly small size compared to the size of bitstream for image data, the bits for the ROI maps were not involved in the performance evaluation of the proposed method. Excising cloud region data when compression could significantly reduce onboard storage and bandwidth requirements. However, the community lacks an accurate algorithm of real-time cloud detection in instrument hardware.

It is meaningful to do onboard compression after cloud detection for high resolution HSI. We focus on the VSWIR electromagnetic spectrum from $0.4-2.5 \mu \mathrm{m}$. There are many studies of cloud detection in these wavelengths, and algorithms vary in their assumptions and complexity. "Classical" cloud screening applies threshold tests to spatial and spectral properties of the image[17]. Pixels whose values fall outside valid ranges are marked as cloudy. For example, the MODIS algorithm compares selected visible and near-infrared (VNIR) and near-infrared (NIR) bands to predetermined thresholds and then aggregates the result in different combinations depending on land type[18][19][20][21]. The algorithm uses a combination of 14 wavelengths and over 40 tests. This underscores the intrinsic difficulty of constructing a universal and complete cloud-screening procedure. Of direct relevance to this work, onboard cloud screening has been demonstrated onboard the EO-1 spacecraft[22]. EO-1 cloud screening uses the solar zenith angle to compute the apparent top-of-atmosphere (TOA) reflectance. Then, it applies a branching sequence of threshold tests based on carefully crafted spectral ratios to distinguish clouds and bright landforms such as snow, ice, and desert sand. The EO-1 cloud detection also acts as a data filtering step prior to onboard cryospheric and flood classification[23][24]. To our knowledge, it is the only previous case of cloud screening performed on orbit. Another kind of onboard cloud detection algorithms are ACCA. They are used to give cloud-cover (CC) predictions to reduce cloud contamination in acquired scenes[25][26][27]. These onboard cloud detecting algorithms are based on threshold in general.

Even more complex algorithms which belongs to ground side are proposed. Some state of-the-art cloud-screening techniques estimate the optical path from absorption features such as the oxygen A band, as in Gómez-Chova et al.[28] or Taylor et al[29]. Thermal infrared (IR) channels can add brightness temperature information. Minnis et al. predict clear-sky brightness temperature values using ambient temperature and humidity and then excise pixels outside these intervals[30]. Texture cues can be also used to recognize clouds by their high spatial heterogeneity[31]. Martins et al. 
demonstrate that a simple spatial analysis, i.e., the standard deviation of VNIR isotropic reflectance in a $3 \times 3$ pixel window, reliably discriminates clouds from aerosol plumes over ocean scenes[32]. Jin hu Bian et al. proposed spectral signature and spatio-temporal context method to distinguish snow from cloud[33]. Markov random field models was developed to segment hyperspectral image. Murtagh et al. represent spatial dependency using a probabilistic Markov random field prior[34]. Haoyang Yu et al. proposed adaptive MRF method combined with SVM and achieve a good performance of classification of terrains[35]. Probabilistic model was another cloud detection method. Gómez-Chova et al. use a Gaussian mixture model to produce posterior probabilities. The Bayesian probabilistic model of Merchant et al. combines observational data with prior predictions from atmospheric forecasts, leading to true probabilistic predictions[36]. David R proposed the decision theoretic method based on Bayesian probabilistic model also that achieved negligible false positives in cloud screening[37]. Recently deep learning are widely used in classification of HSI. Li Wei et al. proposed hyperspectral image classification by using deep pixel-pair features[38]. Bin Pan et al. proposed a kind of vertex component analysis network achieve better performance than some state-of-the-art method[39].

TA method have more false positive that are at high altitudes or at low solar illumination where snow was misclassified as clouds. And the probabilistic model method and the learning-based method result more omission errors that were associated with undetected optically thin clouds over underlying surface because of the incompleteness of training sample for cloud. Our method have good solution for these problems, which is mainly based on exponential spectral angle map, markov random field and dynamic stochastic resonance. The reminder of this paper is organized as follows. Section 2 introduces the difficulties of onboard cloud detection method in detail. The proposed methodology for cloud detection is introduced in Section 3. In Section 4, the proposed method is validated and compared to other cloud detection methods. Section 5 discusses the advantages, limitations and applicability of the proposed method. Section 6 presents the conclusions.

\section{Related work}

Onboard cloud detection almost use the threshold algorithms (TA). Threshold algorithms have difficulty with shadowed cloud or darken snow covered surfaces which are not identified properly in the NDSI test[40]. The used bands of several typical TA methods are shown in table 1. They used $0.66 \mu \mathrm{m}, 0.86 \mu \mathrm{m}, 1.25 \mu \mathrm{m}$ and $1.65 \mu \mathrm{m}$. Since the complexity of various factors, it's nearly impossible to find a classifier plane to separate cloud from ground in spectral feature space, as shown in fig.1.(b). Fig.1.(c) shows that cloud pixels and ground pixels cannot be separated completely under TA classifier due to the overlap of spectral feature. As shown in fig.1.(d), there are some wrong classification in shadowed snow and thin or dark cloud. The yellow region indicated that actual cloud pixels are misclassified into ground under TA method. Contrarily, some snow pixels are misclassified into cloud because spectrum of these snow pixels are mixed with cloud spectrum (see green region).

The influence of clouds on solar irradiance is due to reflection, absorption and scattering of the irradiance by cloud particles and depends strongly on the dimensions, opacity, thickness and composition of the clouds. There are different types of clouds with different dimensions, opacity and properties depending on several parameters, resulting to a different effect on solar irradiance. The clouds are divided in ten types as seen in table 2. Ice crystals and water drops have a different impact at the absorption and scattering of solar radiation especially in SWIR. Fig.2 compares the measured top of atmosphere (TOA) reflectances at SWIR wavelengths. This shows measurements from the Hyperion sensor acquired on 16/4/2011. It also shows three types of cloud that are sampled in one scene and their TOA reflectance curves.

In this scene, water particle effective radii is about $4 \mu \mathrm{m}$ and ice effective radii is $60 \mu \mathrm{m}$. And its illumination is $75^{\circ}$ solar zenith angle. The black curve shows TOA of a piece of thick ice cloud at 10 $\mathrm{km}$ altitude with $0.15 \mathrm{~g} / \mathrm{m}^{3}$ water content overlaying a piece of thin cirrus. The cyan curve shows TOA of an unknown portion of the mixed phase cloud where the ice phase is dominant. The red curve shows TOA reflectance of a piece of water cloud (cumulus) at $5 \mathrm{~km}$ altitude. The three spectral curves 
Table 1. Spectrum used by threshold methods and disadvantage

\begin{tabular}{|c|c|c|}
\hline Method & The used spectrum & Disadvantage \\
\hline ACCA in paper [40] & $\begin{array}{l}0.45-0.52 \mu \mathrm{m}, 0.52-0.6 \mu \mathrm{m} \\
0.62-0.69 \mu \mathrm{m}, 0.76-0.96 \mu \mathrm{m} \\
1.04-1.25 \mu \mathrm{m}, 1.55-1.75 \mu \mathrm{m}\end{array}$ & $\begin{array}{l}\text { They use NDSI index which contains } \\
\text { spectral bands near } 1.65 \mu \mathrm{m} \text { to discriminate }\end{array}$ \\
\hline HCC in paper [22] & $\begin{array}{l}0.55 \mu \mathrm{m}, 0.66 \mu \mathrm{m}, 0.86 \mu \mathrm{m}, 1.25 \mu \mathrm{m}, \\
1.38 \mu \mathrm{m}, 1.65 \mu \mathrm{m}\end{array}$ & $\begin{array}{l}\text { snow and cloud. But some of the shadowed } \\
\text { cloud or darken snow covered surfaces will } \\
\text { be misclassified due to different reflectance }\end{array}$ \\
\hline DCC-ASE in paper [23] & $\begin{array}{l}0.43 \mu \mathrm{m}, 0.56 \mu \mathrm{m}, 0.66 \mu \mathrm{m}, 0.86 \mu \mathrm{m} \\
1.25 \mu \mathrm{m}, 1.38 \mu \mathrm{m}, 1.65 \mu \mathrm{m}\end{array}$ & from various cloud particles. \\
\hline
\end{tabular}

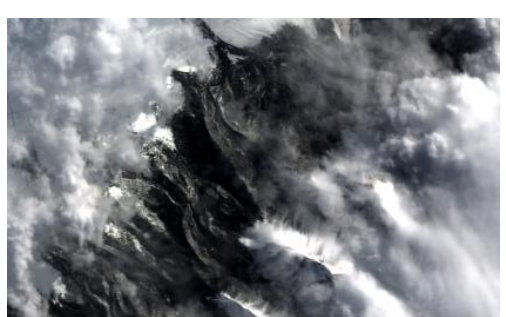

(a)

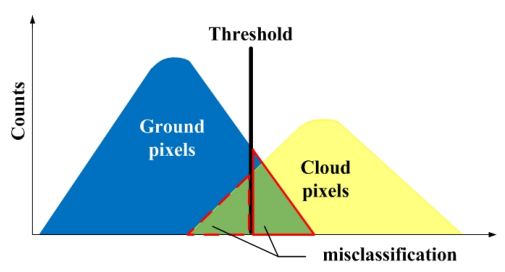

(c)

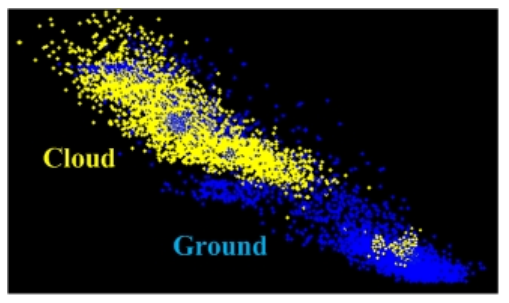

(b)

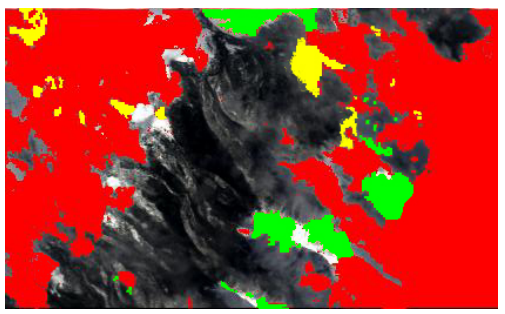

(d)

Figure 1. Cloud detection result under TA method. (a) Original image; (b) Feature space visualization between cloud and ground; (c) Cloud region is detected by threshold algorithm; (d) Diagrammatic sketch of misclassification between ground and cloud pixels under TA method.

have high reflectance, as shown in the top right corner of Fig.2.(b). But the biggest difference of three curves is between $1.5 \mu \mathrm{m}$ to $1.85 \mu \mathrm{m}$. And we can see that liquid cloud has the highest reflectance in this spectral segment. Reflectance of mixed phase cloud is lower and that of ice cloud is lowest. TA have most difficulties in distinguishing ice clouds and snow, then detecting thin or dark cloud. Because spectral feature of snow from NDSI is always overlapping with that of ice cloud. And spectral of thin cloud is affected by underlying surface. The dark cloud have various reflectance due to the changing sun zenith angle or shadowed region.

Most automatic cloud detection methods are based on the radiometric features of clouds. The spectral, frequency, and texture features are the most widely used radiometric features and are extracted from different bands of satellite imagery[41]. According to statistics from 184 scenes of Hyperion level 0.5 data, the solar reflectance of 10 cloud types and different ground types can be seen in electromagnetic spectrum from 0.4-2.5um, as shown in Fig.3. Different clouds may have different amplitude on reflectance. But after normalization, the envelope of the spectral curve are roughly the 
Table 2. Characteristic of 10 types of cloud

\begin{tabular}{ccc}
\hline themodynamic phase & cloud type & \multicolumn{1}{c}{ characteristic } \\
\hline & $\begin{array}{c}\text { Cumulus }(\mathrm{Cu}) \\
\text { Stratocumulus (Sc) } \\
\text { Stratus (St) }\end{array}$ & They are composed of water droplets. \\
Water cloud & Cumulonimbus (Cb) & \\
\hline Mixed phase cloud & Altocumulus (Ac) & They are composed primarily of water droplets; \\
& Nimbostratus (Ns) & however, they can also be composed of ice crystals \\
& Cirrus (Ci) & They temperatures are low enough. \\
\hline Ice cloud & Cirrocumulus (Cc) & can appear in a magnificent array of colors when the \\
& Cirrostratus (Cs) & sun is low on the horizon.
\end{tabular}

same, as shown in Fig.3.(a). But after normalization, different earth surface still have different spectral reflectance, as shown in Fig.3.(b).

The pure threshold method is a simple, efficient, and practical approach for cloud detection, but its sensitivity to the background and the range of cloud coverage make it impractical for general use[42]. Comparing with threshold method, spectral angle map (SAM) have a better cloud detecting performance because of taking advantage of more spectral information. In this paper we demonstrate a cloud-detecting algorithm which mainly combined SAM and MRF. In order to get an accurate cloud cover region, we presented TESAM-aMRF-DSR method for cloud detecting. The following sections describe the algorithm's theoretical method. We then evaluate performance for different operation scenarios by using a decade-year historical image archive of the "classic" Hyperion Spectrometer in section 4 . Then we have a discussion in section 5.

\section{Proposed method}

The MRF have proven to be useful method of enhancing classification accuracy based on spectral and spartial domains separately. Therefore, MRF methods demonstrate promising performance in hyperspectral image classification. To achieve further accuracy improvement, we wish to optimize the features from the spectral and spatial domains simultaneously. To this end, this paper proposes new algorithms that combine TESAM with an adaptive MRF algorithm. The general framework of the proposed methods are shown in fig.4. The hyperspectral images are processed by TA-assisted ESAM algorithm, we obtain the basic classification result. Then aMRF algorithm is the optimal process of TESAM. And DSR is a refinement process for image noise because the data of image is onboard level. After the classification, algorithm compared the spectrum of cloud with the reference spectrum and refresh the reference spectrum.

\subsection{T-ESAM}

SAM measures the angle $\theta(\mathrm{x}, \mathrm{y})$, where $\mathrm{x}$ and $\mathrm{y}$ are $\mathrm{N}$-dimensional spectral vectors having real-valued components, $\left\{x_{i}\right\}_{i=1}^{N}$ and $\left\{y_{i}\right\}_{i=1}^{N}$, respectively,

$$
\theta(x, y)=\arccos \left(\frac{\langle\mathbf{x}, \mathbf{y}\rangle}{\|\mathbf{x}\| \cdot\|\mathbf{y}\|}\right), 0 \leq \theta \leq \frac{\pi}{2}
$$




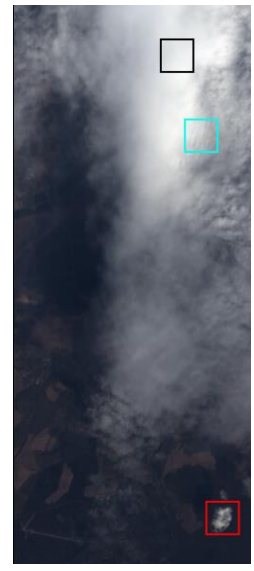

(a)

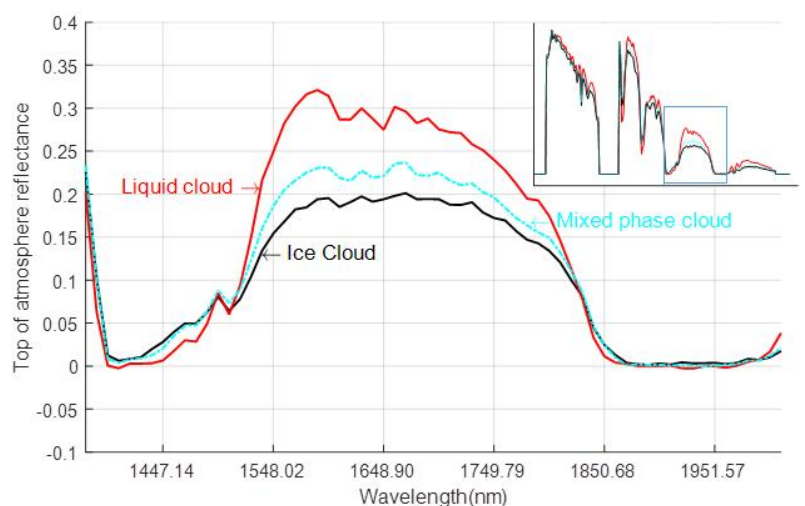

(b)

Figure 2. Black cyan and red line plot top of atmosphere reflectance measurements for liquid and ice clouds in the EO1H1780212011138110Pn scene from Hyperion of EO-1. (a) RGB color of cloud scene with black, cyan and red box. (b) Spectral curve of TOA reflectance from 1.4um to 1.9um among liquid cloud, mixed phase cloud and ice cloud that are from Level 0.5 (on-board processing).

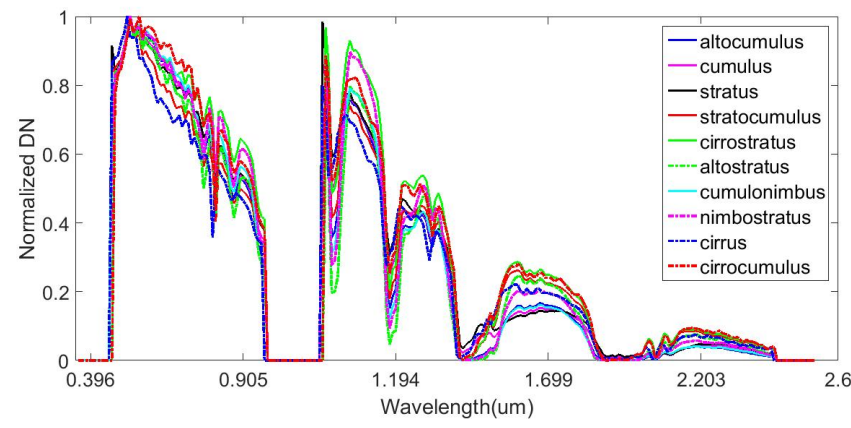

(a)

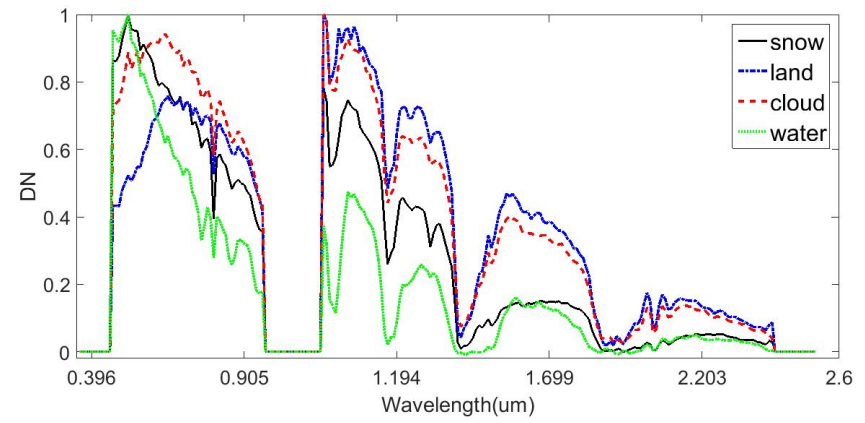

(b)

Figure 3. Spectral curve statistics of cloud and ground reflectance. (a) Normalized spectral reflectance curve of different cloud types. (b) Normalized spectral reflectance curve of different materials.

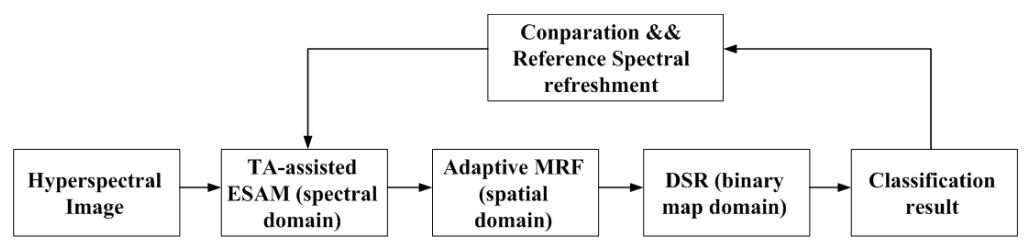

Figure 4. General framework of the proposed methods. 
where $\langle\mathbf{x}, \mathbf{y}\rangle$ is the scalar product between $\mathbf{x}$ and $\mathbf{y}$

$$
\langle\mathbf{x}, \mathbf{y}\rangle=\sum_{i=1}^{N} x_{i} \cdot y_{i}
$$

and $\|\cdot\|$ represents the Euclidean norm, i.e, $\left\|\mathbf{x}^{2}\right\|=\langle\mathbf{x}, \mathbf{x}\rangle$. The $\mathrm{x}$ represents the target spectral vector. And y represents the referenced spectral vector.

Threshold algorithms (TA) for cloud detection onboard such as ACCA algorithm [25] for multispectral and HCC algorithm [22] for hyperspectral appear to be good discriminators for most of the earth. These algorithms can detect cloud roughly accurate. But they have troubles in the variances of sun zenith angle, cloud water content and cloud height. ESAM can be a better measure of similarity for hyperspectral data due to its robustness to illumination variations. We have encapsulated the SAM metric inside an exponential function to produce ESAM function which is a positive semi-definite function and also captures the spectral similarity property of SAM. The ESAM function is defined as

$$
\operatorname{ESAM}(x, y)=\exp (-\theta(\mathbf{x}, \mathbf{y}) \cdot k)
$$

where $\mathrm{k}$ is the gain parameters. ESAM is used to make the cloud region more distinguishable due to amplifying the difference of spectral similarity linearly.

After the 3-D original hyperspectral image $\mathbf{I}_{[\mathbf{L}, \mathbf{W}, \mathbf{H}]}$ processing by ESAM, we can obtain a 2-D computing result. The lowest value of numbers indicate cloud region if there is cloud in image. Simultaneously, threshold algorithms also has detected result of cloud region. Then we can obtain the classifier by combining ESAM with TA.

$$
\begin{aligned}
& \sum_{i=g(\min )}^{g(n)} \operatorname{histogram}(\operatorname{ESAM}(I, y)=i) \leq n_{T A} \\
& \sum_{i=g(\min )}^{g(n+1)} \operatorname{histogram}(\operatorname{ESAM}(I, y)=i) \geq n_{T A}
\end{aligned}
$$

where histogram $(\operatorname{ESAM}(\mathrm{I}, \mathrm{y})=\mathrm{i})$ means histogram statistics of the ESAM results between hyperspectral image and referenced spectrum that equals to " $\mathrm{i}$ ". The $\mathbf{g}(\mathbf{m i n})$ and $\mathbf{g}(\mathbf{n})$ indicate the frequency corresponding to gray level minimum and gray level $\mathbf{n}$ respectively. The $\mathrm{n}_{T A}$ means the pixel-number of cloud region which is detected by threshold algorithms. Then we could obtain a classifier parameter $\mathbf{g}(\mathbf{n})$ that detect the cloud region coarsely when $\mathbf{g ( n )}$ satisfies equation (4) and equation (5) at the same time. The cloud detection coarse classifier is defined as

$$
f(x)=\left\{\begin{array}{l}
c_{1}, \text { if } \operatorname{ESAM}(x, y)<g(n) \\
c_{2}, \text { if } \operatorname{ESAM}(x, y) \geq g(n)
\end{array}\right.
$$

The observed spectrum of instrument data forms a vector $x$ with multiple spectral channels per pixel. The cloud-screen decision maps these pixel brightness values to a binary classification $\mathbf{c}=\mathbf{f}(\mathbf{x})$ : $\mathbf{R}^{d} \rightarrow\{\mathbf{c} 1, \mathbf{c} 2\}$, where c1 represents that there is a cloud present and c2 represents the event that clear sky is observed. Classifier $\mathrm{f}(\mathrm{x})$ detects the cloud coarsely.

\section{2. aMRF model}

The MRF model, which combines spectral and spatial information, is widely used in classification. It can provide an accurate feature representation of pixels and their neighborhoods. The basic principle of aMRF is to spatial correlation information into the posterior probability of the spectral features. 


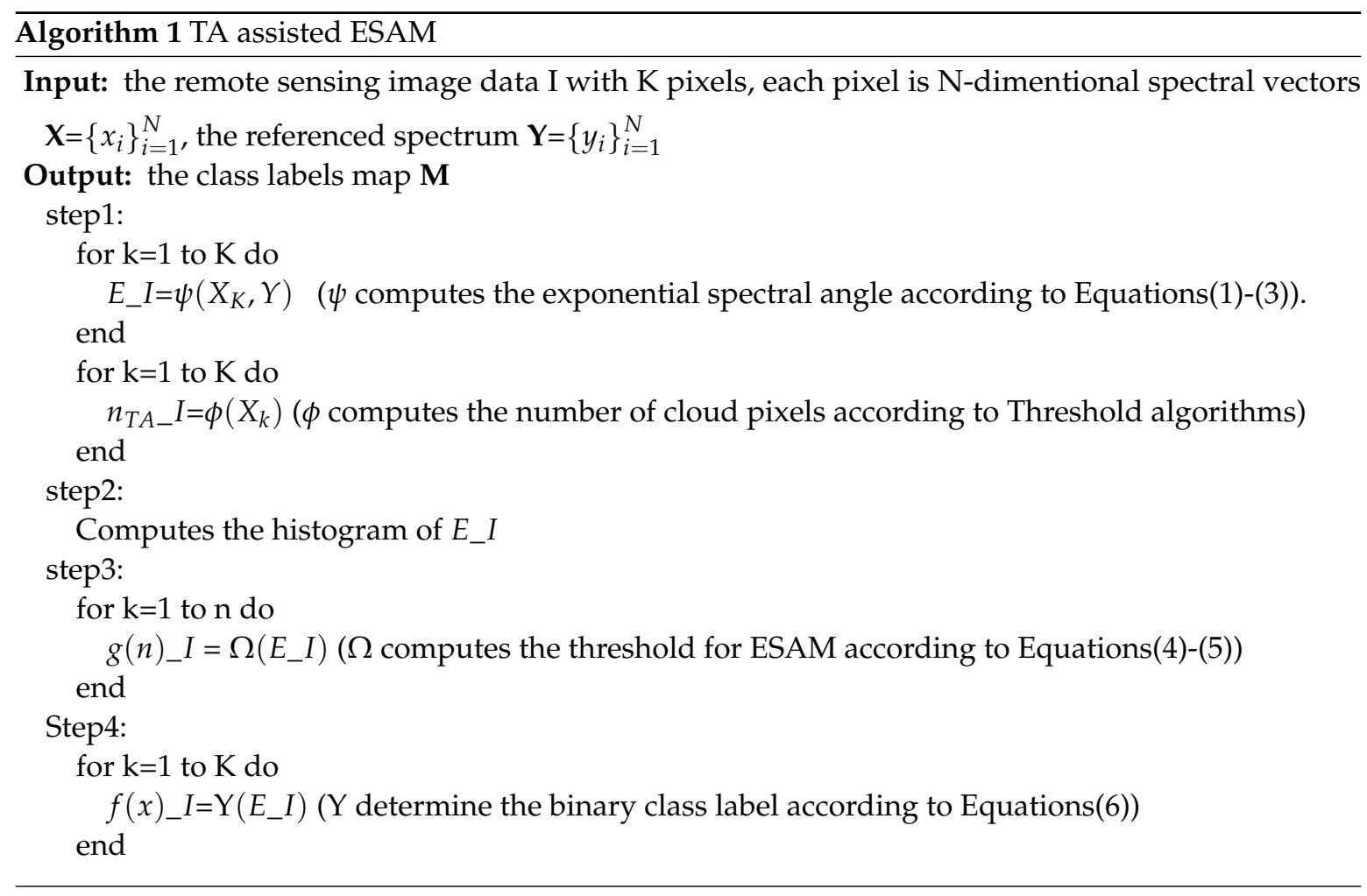

Based on the maximum posterior probability principle, the classic MRF model can be expressed as follows:

$$
p\left(x_{i}\right)=-\frac{1}{2} \ln \left|\Sigma_{K}\right|-\frac{1}{2}\left(x_{i}-m_{k}\right)^{T} \Sigma_{K}^{-1}\left(x_{i}-m_{k}\right)-\beta_{i} \sum_{\alpha i}\left[1-\delta\left(\omega_{k i}, \omega_{\alpha i}\right)\right]
$$

Where $m_{k}$ and $\Sigma_{K}$ are the mean vector and covariance matrix, respectively, of class $\mathrm{k}$ and the neighborhood and class of pixel $i$ are represented by $\alpha_{i}$ and $\omega_{k}$, respectively. In this paper, we separate the pixels of remote sensing image into 2 classes by equation (6), ground pixels and cloud pixels. The parameter $\beta_{i}$, called the weight coefficient is used to control the influence of the spatial term.

To obtain the local spatial weight coefficients $\beta_{i}$, Haoyang Yu[43] etc. used the noise-adjusted principal components (NAPC) transform to obtain the first principal component to calculate the $\beta_{i}$,

$$
\beta_{i}=\beta_{0} \cdot R_{H} I_{i}=\beta_{0} \cdot \frac{v^{a r_{k}}}{\operatorname{var}_{i}}
$$

where var $_{k}$ represents the class-decision variance of the neighborhood of pixel $\mathrm{i}$ as determined by majority voting rules and $v a r_{i}$ is the local variance of pixel i [44]. When $R H I_{i}$ is high, it can be concluded that pixel $\mathrm{i}$ is located in a homogeneous region. By contrast, pixel $\mathrm{i}$ is on a boundary when $R H I_{i}$ is low. spatial weight coefficient when var $_{i}=$ var $_{k}$; usually, $\beta_{0}=1$.

According to Equation (7), the aMRF model can be devided into two components: the spectral term and the spatial term. Thus, Equation (7) can be represented in the form

$$
p\left(x_{i}\right)=a_{i}(k)+\beta_{i} \cdot b_{i}(k)
$$

where $\delta\left(\omega_{k i}, \omega_{\alpha i}\right)$ is the Kronecker delta function, defined as

$$
\delta\left(\omega_{k i}, \omega_{\alpha i}\right)=\left\{\begin{array}{l}
1, \omega_{k i}=\omega_{\alpha i} \\
0, \omega_{k i} \neq \omega_{\alpha i}
\end{array}\right.
$$




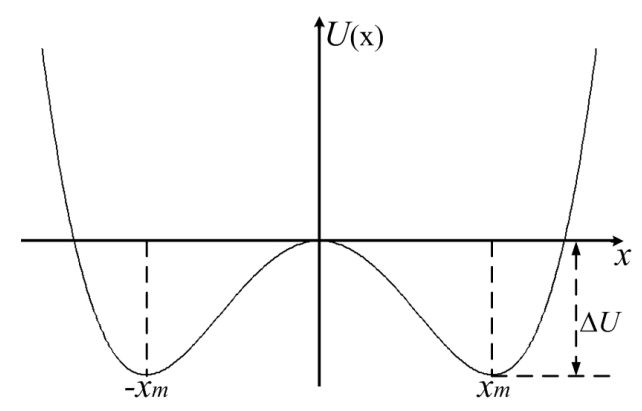

Figure 5. SR in double-well potential valley.

The pseudocode for the TESAM algorithm combined with the aMRF algorithm, abbreviated as TESAM-aMRF, is shown in Algorithm 2.

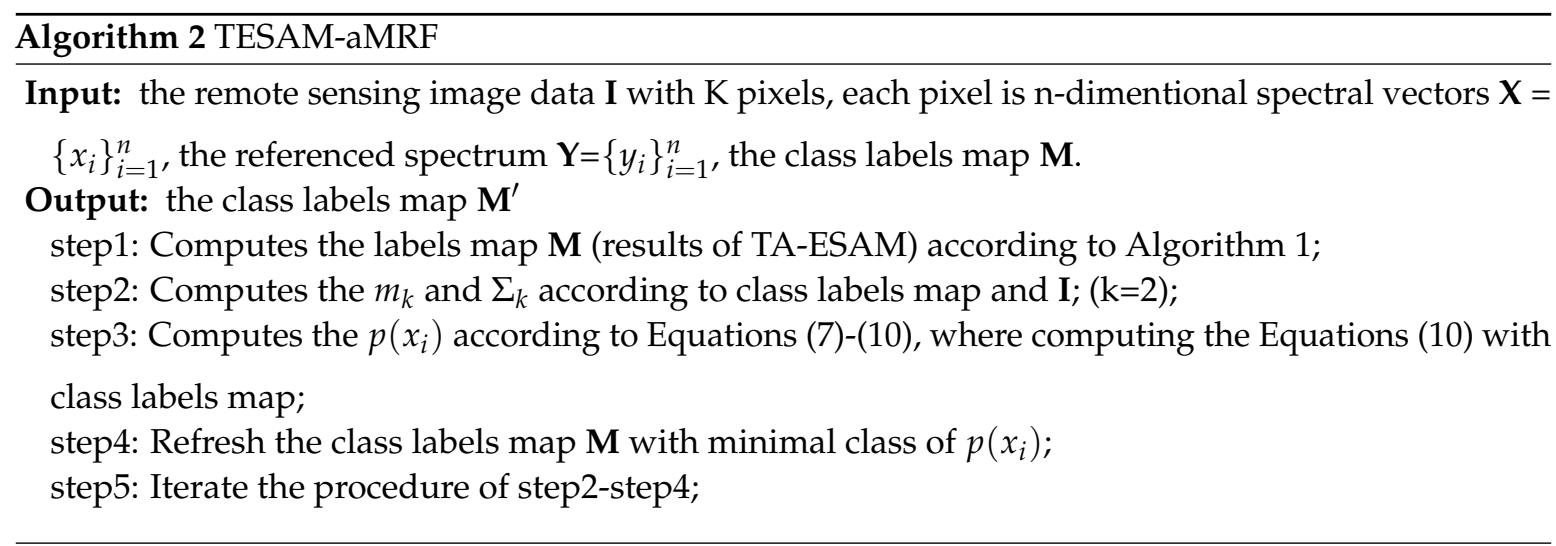

\subsection{DSR model}

The bistable-SR model conventionally used by the physicists shall be explored and elaborated for application to contrast enhancement of a digital image. In analogy to Benzi's double-well model to explain ice ages, the image pixel value is treated like a discrete kinetic parameter, say, the position of a particle in the double well. For a low-contrast image, the analogy states that the pixel is initially in a weak signal state (because of low-intensity value since image is low contrast, i.e. a subthreshold signal). Addition of optimum amount of noise effects its transition to the strong signal state, just as a particle makes a transition from one well to another. Such a change of state of pixel under noise can be modelled by Brownian motion of a particle placed in a double-well potential system shown in Fig. 5 .

A classic 1D nonlinear dynamic system that exhibits SR is modelled with the help of the Langevin equation of motion is given below

$$
m \cdot \frac{d^{2} x(t)}{d t^{2}}+\gamma \cdot \frac{d x(t)}{d t}=-\frac{d U(x)}{d x}+\sqrt{D} \cdot \xi(t)
$$

This equation describes the motion of a particle of mass $m$ moving in the presence of friction, $\gamma$. The restoring force is expressed as the gradient of some bistable potential function $\mathrm{U}(\mathrm{x})$. In addition, there is an additive stochastic force $\xi(t)$ of intensity D.

If the system is heavily damped, the inertial $m \frac{d^{2} x(t)}{d t^{2}}$ term can be neglected. Rescaling the system in (11) with the damping term $\gamma$ gives the stochastic overdamped Duffing equation, which is frequently used to model non-equilibrium critical phenomena as given in (12)

$$
\frac{d x(t)}{d t}=-\frac{d U(x)}{d x}+\sqrt{D} \cdot \xi(t)
$$


where $\mathrm{U}(\mathrm{x})$ is a bistable quartic potential given in

$$
U(x)=-a \cdot \frac{x^{2}}{2}+b \cdot \frac{x^{4}}{4}
$$

Here, $\mathrm{a}$ and $\mathrm{b}$ are positive bistable double-well parameters. The double-well system is stable at $x_{m}= \pm \sqrt{\frac{a}{b}}$ separated by a barrier of height $\Delta U=\frac{a^{2}}{4 b}$ when $\xi(t)$ is zero. The Langevin equation describes the motion of particle in a general double-well.

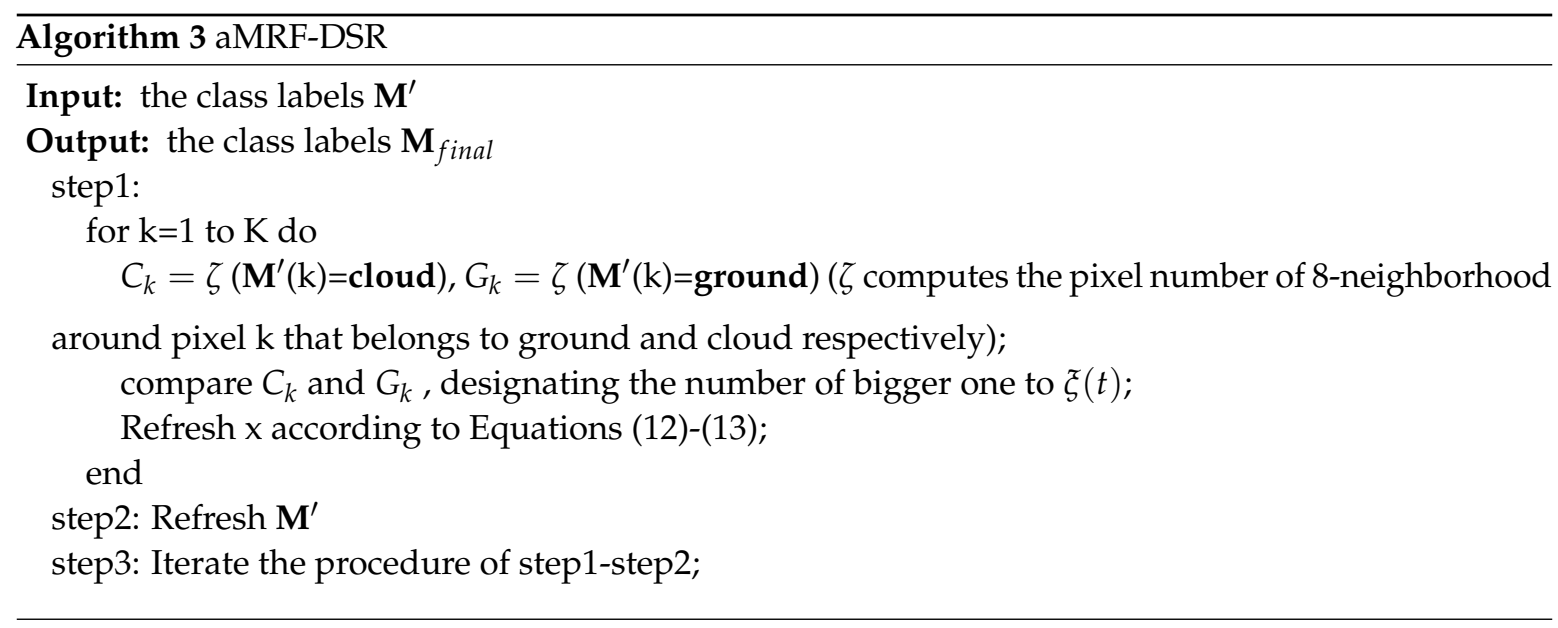

\section{Method Feasibility Validation}

\subsection{Dataset}

In this section, we evaluate the performance of the proposed algorithms using the widely used hyperspectral data from hyperion sensor. The data are Level 0.5 that are used in onboard processing. And they are downloaded from USGS website. Terrains of the dataset contain city, ocean, forest, mountain range, desert, snow and cryosphere. And time span contains spring, summer, autumn, winter, morning, noon and dusk of recent decade year. The span of latitude contains tropical, substropical, temperate, frigid and polar zone. Geographical distribution of the selected scenes are spread all over the world. And season distribution are at all seasons but mainly focusing on winter. Statistics of test dataset is shown in Fig.6.

\subsection{Accuracy Accessment}

The following three different accuracies measures, which were the overall accuracy, precision and recall, were used to assess the accuracy of the algorithm results. Define the True Positive (TP) as the number of clouds correctly labeled as belonging to clouds in the algorithm, False Negatives (FN) as the number of clouds incorrectly labeled as belonging to none-clouds, and True Negatives (TN) as number of none clouds which also labeled as belonging to none clouds. The accuracy, precision and recall are then defined as

$$
\begin{gathered}
\text { Recall }=T P /(T P+F N) \\
\text { Precision }=T P /(T P+F P) \\
F P R=F P /(F P+T N)
\end{gathered}
$$

In the cloud case, precision denotes the proportion of truly cloud pixels in the cloud detection results, while recall is of all pixels that are actually clouds in the image, what fraction of them were 


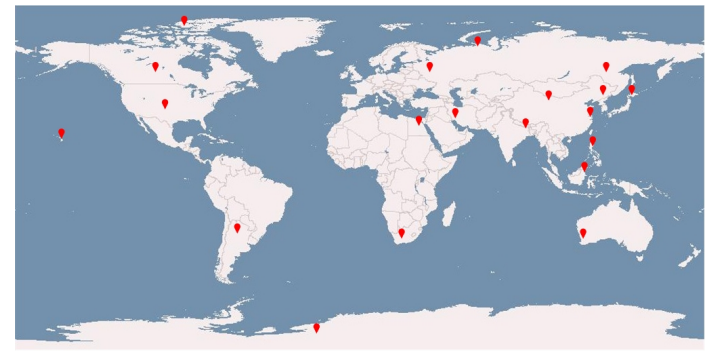

(a)

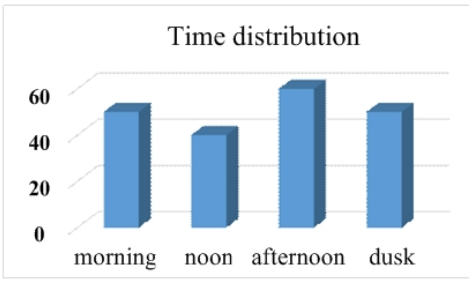

(c)

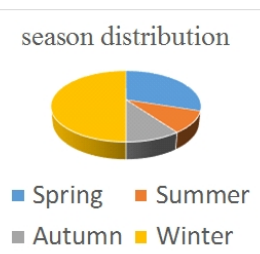

(b)

\begin{tabular}{|c|c|c|c|c|}
\hline & \multicolumn{2}{|c|}{ Ordinary Terrain } & \multicolumn{2}{c|}{ Special Terrain } \\
\hline Terrain & City & Forest & Snow & Cryosphere \\
\hline number & 18 & 17 & 28 & 21 \\
\hline Terrain & Field & Mountain & Desert & Ocean \\
\hline number & 29 & 36 & 17 & 18 \\
\hline
\end{tabular}

(d)

Figure 6. Test dataset description. (a) Geographical distribution of the selected scene; (b) Season distribution of the selected scene; (c) Time distribution of the selected scene; (d) Scene number of each terrain.

detected as clouds. For precision and recall, they are better reflects the errors of cloud classification than overall accuracy.

\subsection{Detection results}

Figure 7 depicts the cloud detection results for different terrain. By visually comparing the results with the false color composites, it is clear that the algorithm developed in this study can accurately separate the cloud pixels. Fig.7.(a) was a summer image acquired at $8^{\text {th }}$ August 2013 with cirrostratus over the desert. The detection result indicate that algorithm showed its strong ability in excluding the cloud pixels from desert pixels even if cloud is such thin that spectrum of cloud is mixed with spectrum of desert pixels. On the other hand fig.7.(b) was a winter image acquired at $3^{r d}$ June 2013 with dark stratus over the ocean and coast. Cloud contain water droplets that have the same materials of ocean in this season. But water of the ocean is in liquid, and that of cloud in gaseous. Spectrum of the same materials is different due to different forms and temperature. The ground of fig.7.(c) and fig.7.(d) are both mountain range. Fig.7.(c) was a spring image acquired at noon on $22^{\text {th }}$ May 2012 with cumulus and stratocumulus around Himalaya mountains. And fig.7.(d) was a winter image acquired at dusk on $3^{\text {rd }}$ January 2007 with altocumulus over mountain. Comparing fig.7.(c) with fig.7.(d), we could see that cloud of the former is lighter than the later because the sun zenith angle of fig.7.(c) is smaller. But both the two image have good cloud detection result even if the dark cloud can be also detected accurately. Fig.7.(f) was a winter image acquired at $28^{\text {th }}$ March 2005 with cumulus over the city Haerbin. Not only the freezing river and the city highlights weren't be classified into cloud pixels. But these cloud that floating over the frozen river are separated from ground successfully. And the thin dark cloud which is in the shadowed region is detected accurately without misclassifying the light ground pixels into cloud. Fig.7.(e), fig.7.(i) and fig.7.(j) are both clouds over the snow or ice. Fig.7.(e) was an image acquired at $12^{\text {th }}$ May 2012 with stratocumulus over snowfield in the cryosphere. Some pices of clouds that are undistinguishable by eyes are floating over the snow field. These cloud pixels are detected correctly. Fig.7.(i) was a spring image acquired at $17^{\text {th }}$ March 2007 with altostratus over mountain which is covered by snow. The edge of altostratus doesn't have a clear outline under visible spectrum and it looks similar with ground. And there are also some cloud pixels that are hard to be distinguished by eyes. Detection result show that unobvious easily confused clouds were also 

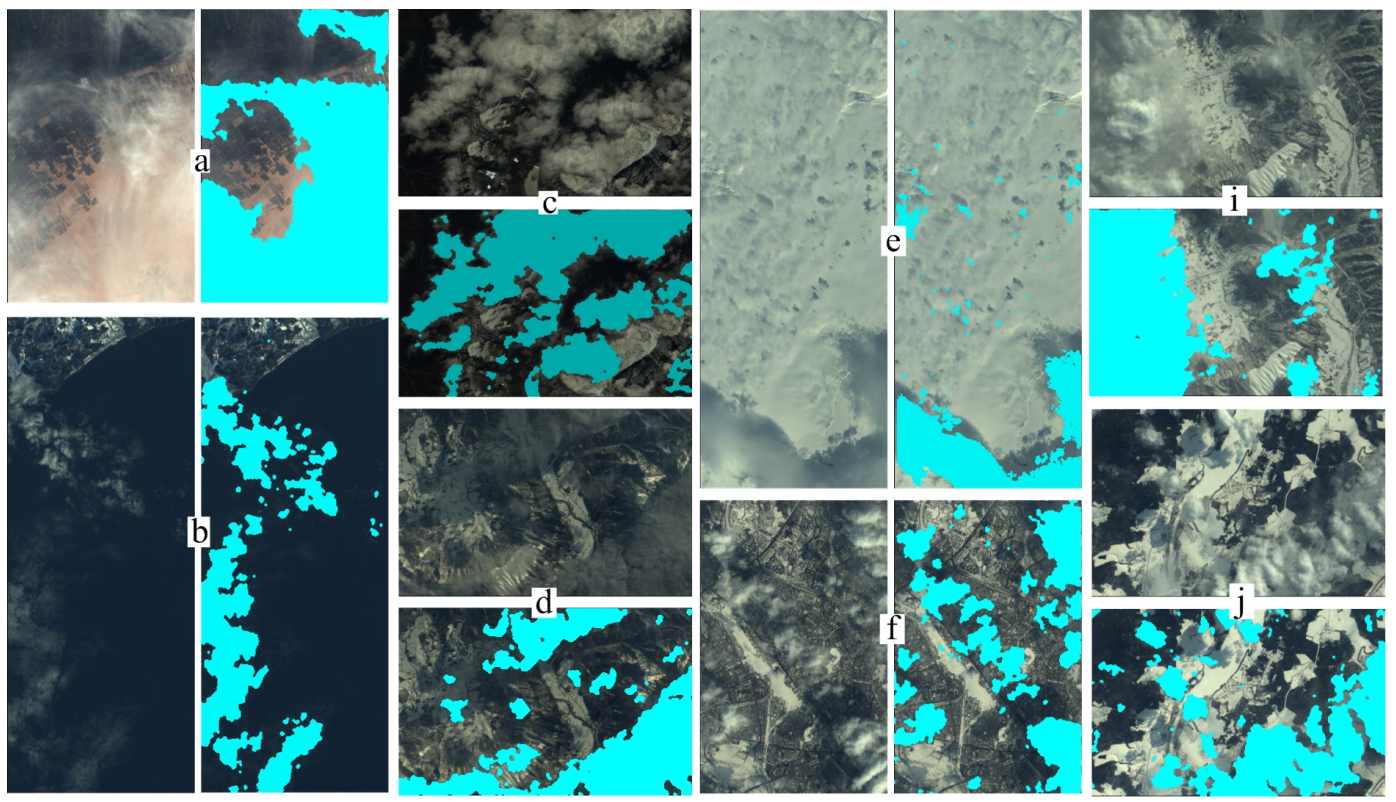

Figure 7. Cloud separation results for different kinds of ground. (a) Desert with thin cloud scene and cloud detection result; (b) Ocean scene and cloud detection result; (c) Mount Qomolangma scene and cloud detection result; (d) Mountain with dark cloud scene and cloud detection result; (e) Snow cover scene and cloud detection result; (f) Highlight city with frozen lake scene and cloud detection result; (i) Mountain with thin cloud scene and cloud detection result; (j) Frozen field scene and cloud detection result.

accurately identified through the proposed method. Fig.7.(j) was a spring image acquired at $28^{\text {th }}$ March 2005 with cumulus over the forest which is cover by some frozen lake. In this image, most of the cumulus is floating on the ice. And they are detected accurately.

\subsection{Performance of Each Stage for the Cloud Detection}

Fig. 8 provides an illustration of the algorithm performance for the each processing stage. It depicts the cloud results for EO-1 Hyperion images from four different dates. By visually comparing the results with the false color composites, it is clear that the algorithm developed in this study can accurately separate the cloud pixels. Threshold algorithm, the traditional method, detects the relatively darker cloud. But there were some FN classification in light cloud region due to various reflectance under threshold algorithm because of the unsuitable parameter. Contrarily, TESAM could accurately detect the thick and light cloud region which is misclassified under threshold method, as shown in Fig.8.(h) and Fig.8.(i). And under TESAM, the various reflectance didn't have much influence over cloud detection. Comparing with threshold method, TESAM seems to be conservative, abstaining from ambiguous classification to prevent mixture of heterogeneous spectrum for aMRF procedure. The ambiguous classification is shown in the yellow circle of fig.8.(k), but these region are not labeled as cloud in fig.8.(1). After TESAM detecting, the cloud region detected by TESAM work as a role of seed region in the procedure of aMRF. By comparing yellow circles of Fig.8.(d) and Fig.8.(e), we can see that after aMRF detecting, some cloud region growed more full. And TN regions have been recovered to ground pixels because aMRF have fault-tolerant ability by combining spectral and spatial information. The detail iteration procedure of aMRF will be introduced later. But the spectrum of some individual pixels has strong similarity with cloud under the selected bands for aMRF. Hence, even if considering the neighbors' contribution, the energy of these pixels under aMRF algorithm are still very low. They were misclassified as cloud pixels. However, these pixels in mask were seemed as noise for DSR. By comparing fig.8.(m) and fig.8.(n), DSR turned these binary properties of "noisy point" over. 


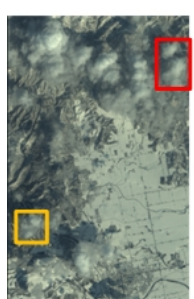

(a)

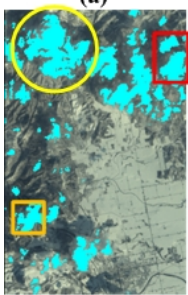

(d)

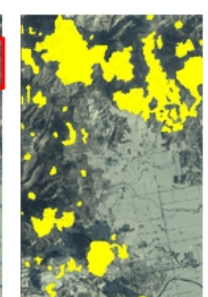

(b)

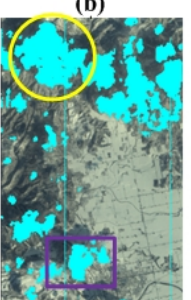

(e)

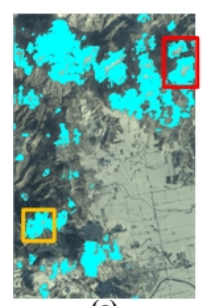

(c)

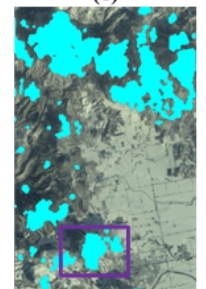

(f)

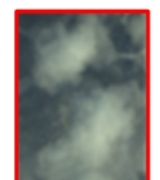

(g)

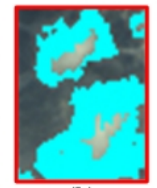

(h)

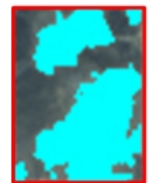

(i)

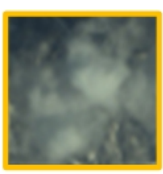

(j)

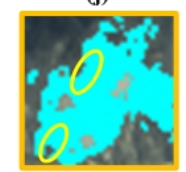

(k)

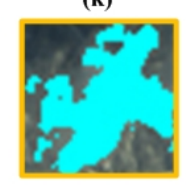

(I)

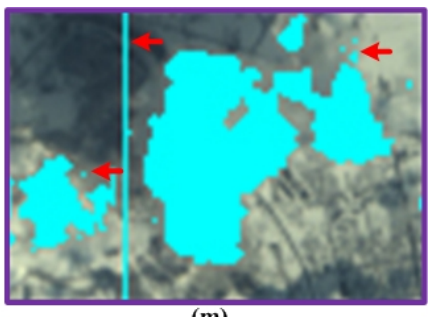

(m)

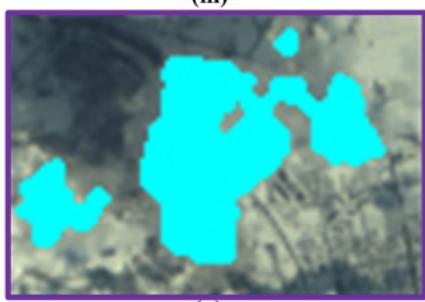

(n)

Figure 8. Comparation of cloud detection results. (a) A winter image acquired at 7 December 2013 with obvious clouds over the whole image. (b) Result of manually labeled image. (c) Cloud detection result by using threshold method. (d) Cloud detection result by using TESAM method. (e) Cloud detection method by using aMRF method, which is based upon (d). (f) Cloud detection method by using DSR, which is based upon figure $8 \mathrm{e} .(\mathrm{g}),(\mathrm{h})$ and (i) are origin picture, TA labeled and TESAM labeled of cloud region respectively that are corresponding to the red box in (a), (c), (d). Picture (j), (k) and (l) are corresponding to origin picture, TA labeled and TESAM labeled of cloud region respectively that are corresponding to the orange box in (a), (c), (d). (m) is the result of aMRF method, which is corresponding to purple box of (e). (n) is labeled by DSR based on (e), which is corresponding to purple box of (f).

The vertical line and some isolate pixels in fig.8. $(\mathrm{m})$ have been eliminated after DSR processing, as shown in fig.8.(n). By comparing fig.8.(b) and fig.8.(f), algorithm detecting result is high consistent with manual labeling result.

The detailed example for iterative process of aMRF is shown in Fig.9. The cloud region which are detected by TA method and TESAM are rather limited. Only a few detected cloud pixels existed in the mask, as seen in Fig.9.(a) and (b). The detection result is treated as initial classification for aMRF. Comparing fig.9.(c-h), we can see that aMRF method have strong robustness if the spectrum of initial seed region is pure enough. And after 8th iteration, classification has good agreement with the real cloud region. And this image tended to be convergent at the 16th iteration.

Fig.10 shows cloud detection performance comparision of each terrain. Terrain from the first row to last row are ocean, mountain, city, desert, ice and cryosphere. It can be seen that the proposed

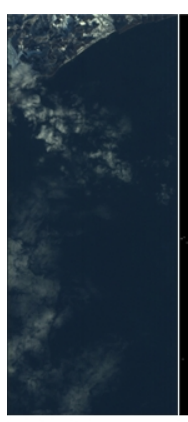

(a)

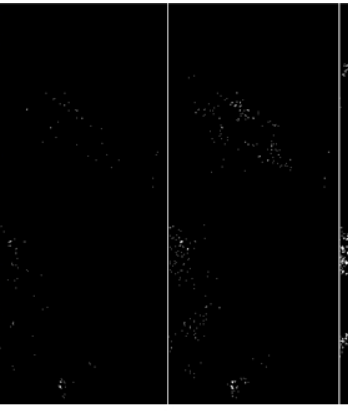

(b)

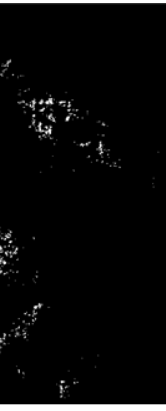

(d)

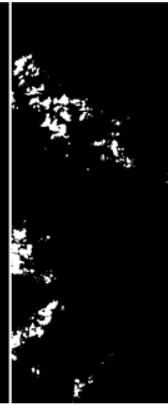

(e)

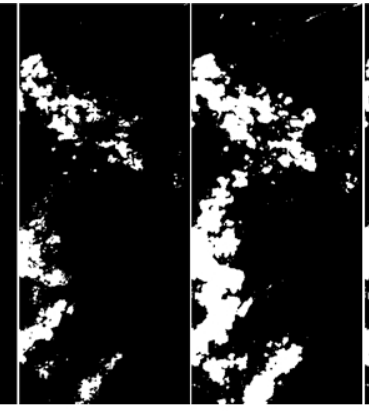

(g)

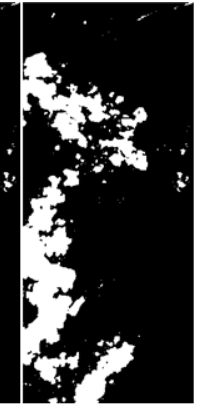

(h)

Figure 9. Comparation of aMRF iteration result. (a) Original image; (b) Classification result of TESAM; $(\mathrm{c}-\mathrm{h})$ The $1^{\text {st }}, 2^{\text {nd }}, 4^{\text {th }}, 6^{\text {th }}, 8^{\text {th }}, 16^{\text {th }}$ and $30^{\text {th }}$ iteration result respectively. 


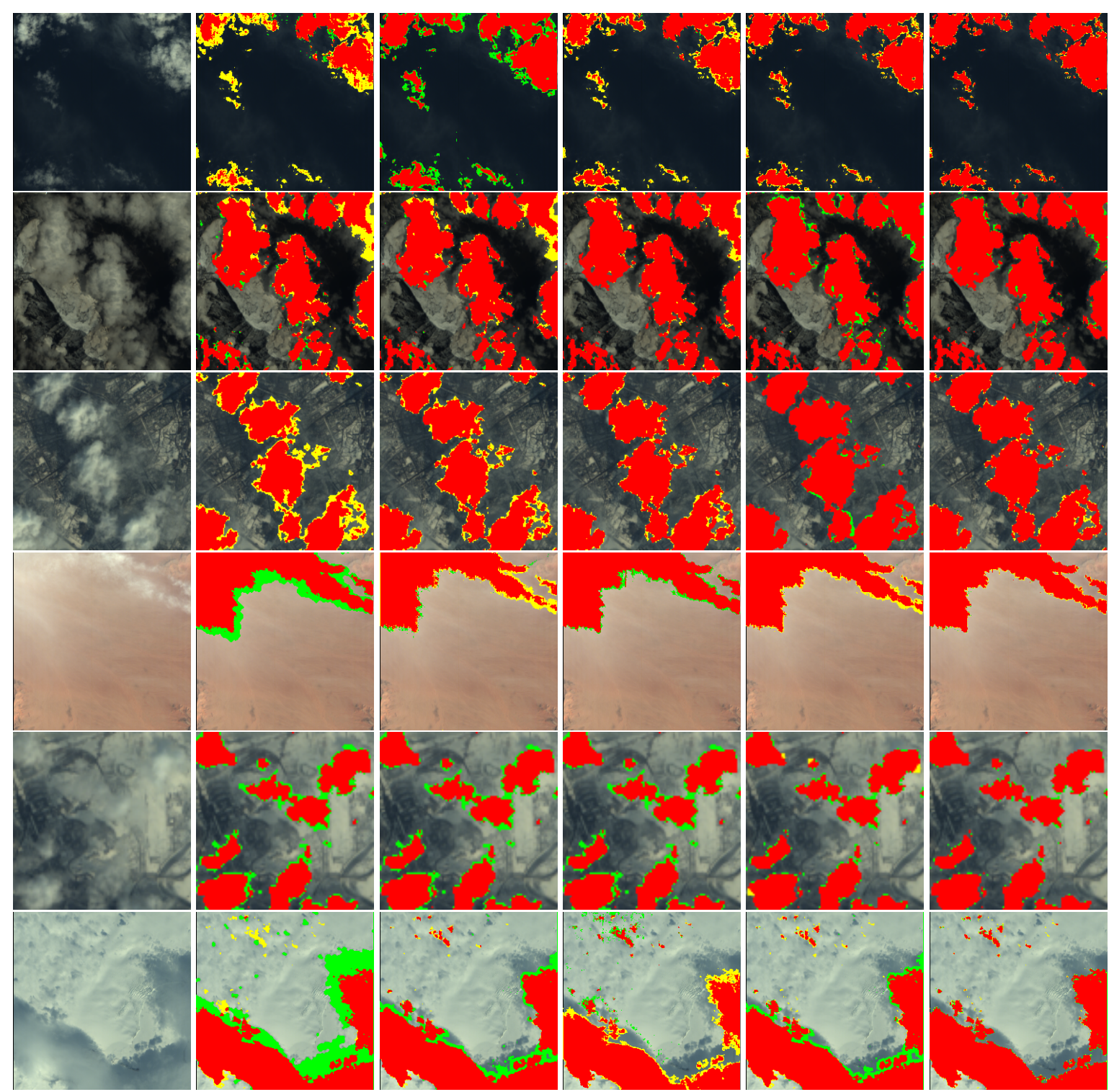

Figure 10. Cloud detection performance comparasion (Red denotes extracted true cloud regions, yellow denotes missed true cloud regions and green denotes non-cloud regions misjudged as cloud regions).

method produced the best precision ratio and recall ratio and its error was lower than that of the other methods. ACCA have high FN under ordinary terrain and high FP under special terrain due to lacking thermal infrared band. HCC have trouble in detecting thin or dark cloud and these scenes that have the same particles to cloud. The support vector machine adaptive markov random field (SVM-aMRF) and rolling guidance filter and vertex component analysis network(R-VCANet) have higher recall ratio and precision ratio than the previous two. But they still have some classification error for thin cloud that mainly because thin clouds are mixed with other spectrum that can't be learned sufficiently. ROC curve and precision and recall curve can be seen in fig.11 and fig.12.

\section{Discussions}

\subsection{The Effectiveness of Combining Threshold Algorithm and Spectral Angle Map}

Spectral Angle Map is widely used due to its simplicity and geometrical interpretability. SAM is invariant to (unknown) multiplicative scalings of spectra due to differences in illumination and angular 


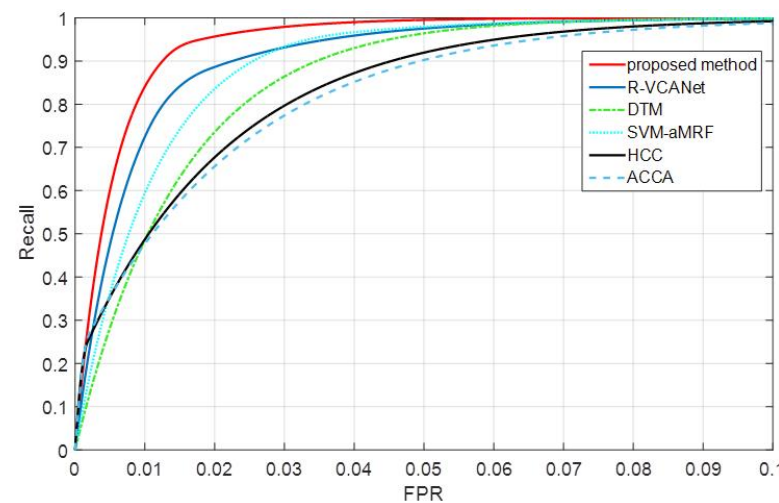

(a)

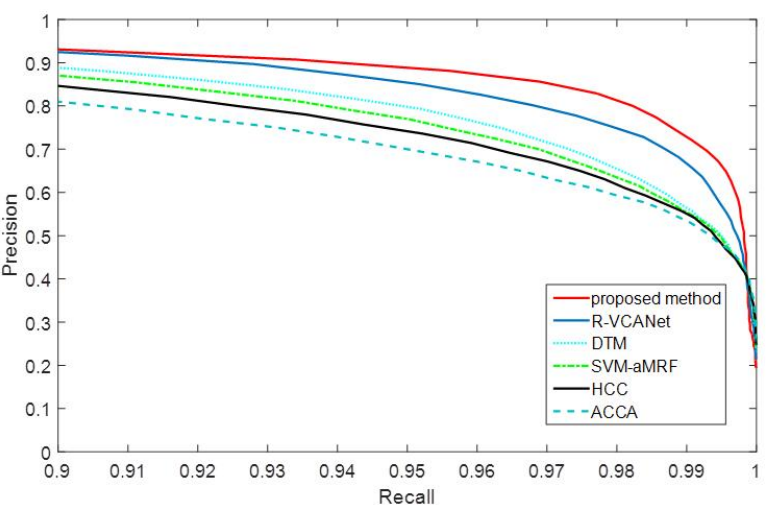

(b)

Figure 11. (a)ROC curve of cloud detection performance under each method. (b)Precision performance curve corresponding to Recall under each method.

orientation. One of the most important properties of the spectral-angle distance is the invariance to multiplicative scaling. Due to the invariant nature of the angle among the linearly scaled variations, the spectral angle between two pixels is more sensitive to the pattern (shape) of the spectral signatures instead of absolute intensities. Traditional TA method sometimes overestimate or underestimate the cloud region because the fixed parameters were inappropriate to the variant illumination and angular orientation. In theory, TESAM method could reduce the misclassification error maximatily.

\subsection{The Usefulness of Spatial Contextual Information for Cloud Detection}

Spatial information to the repeated local patterns and their regular arrangement of the ground objects in the images. The spatial information can better reflect the macroscopic properties and the detail structures of the ground objects than a single pixel. Correctly understanding the texture difference of clouds and ground can provide the important basis for their discrimination. For hyperspectral image, texture feature is not obvious enough to discriminate cloud from ground sometimes, especially from snow or highlight features. But the classification result could be very noisy if spatial information is not taken into account. To our knowledge, the spatial information of clouds is a type of random texture. Even though the texture element of clouds is variable and unpredictable, it is still have some different from the texture feature of ground. Markov random field is a popular tool to exploit the spatial information in the classification of hyperspectral data. The aMRF was used to synthesize all the spectral and spatial information into an energy index to find the class attribute at the regional scales. The aMRF is a probabilistic model that is used to integrate spatial information into image classification. With the help of spatial contextual information, the combination of TESAM and aMRF significantly outperforms the TA, probabilistic methods and deep learning methods. The performance of the aMRF was robust regardless of snow, highlight ground and shadowed cloud. In general, it achieved the best performance when the energy was stable, then the iteration cloud be terminated.

\subsection{Error Sources of the proposed Method}

In brief, the cloud detection results indicated that the proposed method have a good performance for the detecting cloud in EO-1 images. However, several error sources which might influence the algorithm accuracy should also be pointed out. The first error source came from the way which the cloud region detected by TA algorithm bigger than their actual size due to unsuitable parameters. Correspondingly, TESAM will overestimate cloud areas because the cloud region size is depended by histogram of TESAM result and TA result together. In this condition, it will increase FPR region of 
TESAM results, which may lead to error classification of large area during aMRF processing due to impure cloud spectrum.

\subsection{Applicability of the Developed Methods in the Feature}

The proposed method is highly automatic and efficient when processing a tremendously large volume of imagery real-time. It can be easily implemented on a parallel processor, such as FPGA. The proposed method needs some external storage devices and architectures such as ping-pong structure which could restore data for supporting the use of spatial context. Moreover, classifiers instantiated in hardware logic typically have already achieved in the implementation of arccosine[45], exponentials[46][47] functions, and even floating-point operations, supporting many nonlinear classifiers and naive implementations of linear classifiers. Additionally, there are real-time requirements for processing procedure. Bandwidth of multi DDRs could satisfy $\mathrm{Gb} / \mathrm{s}$ throughput of algorithm by using a small fixed number of arithmetic operations on locally available data. It also can be adapted for images acquired by similar satellite instruments, which have similar spectral bands and temporal resolutions. The method in this paper is general and efforts in the future will be put into the test for other regions with different environments.

\section{Conclusions}

Accurate detection of clouds for satellite images is the fundamental pre-processing step for a variety of remote sensing applications. This paper presented a new pratical method for cloud discrimination by combining from many past approaches and intergrating the spectral signatures with spatial information. The methodology included three closely related major stages, initial spectral classification stage, spatial-spectral classification stage and noise elimination stage.

Visual accessment revealed that the method developed in this paper can accurately separate the cloud and ground pixels. The pixel-level accuracy accessment was performed by comparing the detection results with manually labeled image. Good agreements were found between detection results and manually labeled image with the average overall accuracy $96.28 \%$. The spatial information can approximately exclude misclassified $8.35 \%$ cloud pixels from initial spectral tests. Generally, the proposed method exhibited high accuracy for clouds discrimination of EO-1 Hyperion images and was an improvement over the traditional spectral-based algorithms. It can provide an accurate cloud mask for the on-going EO-1 images and the similar satellites with the same temporal and spectral settings.

Acknowledgments: Project supported by the National Nature Science Foundation of China(No.60543006). The authors would like to thank the editor and reviewers for their instructive comments that helped to improve this manuscript. Besides, they would also like to thank the international scientific data service platform and the U.S. Geological Survey website

Author Contributions: Haoyang Li, Hong Zheng and Chuanzhao Han conceived of the study and designed the experiments; Haibo Wang and Min Miao took part in the research and analyzed the data; Haoyang Li wrote the main program and most of the paper.

Conflicts of Interest: The authors declare no conflict of interest.

\section{Abbreviations}

The following abbreviations are used in this manuscript: 


$\begin{array}{ll}\text { HSI } & \text { Hyperspectral image } \\ \text { TESAM } & \text { Threshold assissted exponential spectral angle map } \\ \text { aMRF } & \text { Adaptive Markov Random Field } \\ \text { DSR } & \text { Dynamic Stochastic Resonance } \\ \text { TA } & \text { Threshold algorithm } \\ \text { NDSI A } & \text { Normalized Difference Snow Index } \\ \text { TOA } & \text { Top of Atmosphere } \\ \text { ACCA } & \text { Automatic Cloud Cover Algorithm } \\ \text { HCC } & \text { Hyperion Cloud Cover } \\ \text { DCC-ASE } & \text { Detection of Cryospheric Change Automonous Sciencecraft Experiment } \\ \text { EO } & \text { Earth Observation } \\ \text { SWIR } & \text { short-wave infrared } \\ \text { SVM-aMRF } & \text { Support Vector Machine adaptive Markov Random Field } \\ \text { R-VCANet } & \text { Rolling Guidance Filter and Vertex Component Analysis Network } \\ \text { ROC } & \text { Receiver Operating Characteristic Curve }\end{array}$

\section{References}

1. Hongda Shen; W. David Pan; Dongsheng Wu, Predictive lossess compression of regions of interest in hyperspectral images with no-data regions. IEEE Trans. Geosci. Remote Sens. 2016 55, 173-182.

2. Hongda Shen; W. David Pan; Dongsheng Wu, Predictive Lossless Compression of Regions of Interest in Hyperspectral Image Via Correntropy Criterion Based Least Mean Square Learning. In proceedings of IEEE International Conference on Image Processing, Phoenix, Arizona, USA, 25-28 Sept 2016.

3. L. Mandrake; C. Frankenberg; C. W. O’Dell; G. Osterman; P. Wennberg; D. Wunch, Semi-autonomous sounding selection for OCO-2. Atmosp. Meas. Tech. Discuss.2013, 6, 5881-5922.

4. L. S. Chien; D.Mclaren; D. Tran; A. G. Davies; J. Doubleday; D. Mandl, Onboard product generation on earth observing one: A pathfinder for the proposed HyspIRI mission intelligent payload module. IEEE JSTARS. 2013, 6, 257-264.

5. Marco Conoscenti; Riccardo Coppola; Enrico Magli, Constant SNR, Rate Control, and Entropy Coding for Predictive Lossy Hyperspectral Image Compression. IEEE Trans. Geosci. Remote Sens. 2016, 54, 7431-7441.

6. M. Klimesh; A. Kiely; and P. Yeh, Fast lossless compression of multispectral and hyperspectral imagery. In proceedings of 2nd Int. Workshop Onboard Payload Data Compress. Noordwijk,Netherlands, 1-8 Sept 2010.

7. N. Aranki; A. Bakhshi; D. Keymeulen; M. Klimesh, Fast and adaptive lossless on-board hyperspectral data compression system for space applications. In Proc. IEEE Aerosp. Conf. Montana, USA, 7-14 March 2009.

8. D. Keymeulen; N. Aranki; B. Hobson; A. Kiely; M. Klimesh; K. Benkrid, GPU lossless hyperspectral data compression system for space applications. In Proc. IEEE Aerosp. Conf. Montana, USA, 3-10 March 2012.

9. J. Gonzalez-Conejero; J. Bartrina-Rapesta; J. Serra-Sagrista, JPEG 2000 encoding of remote sensing multispectral images with no-data regions. IEEE Geosci. Remote Sens. Lett. 2010, 7, 251 - 255.

10. R. Eastman; S. Warren; C. Hahn, JPEG 2000 encoding of remote sensing multispectral images with no-data regions. Variations in cloud cover and cloud types over the ocean from surface observations, 1954-2008. J. Climate. 2011, 24, 5914-5934.

11. M. Mercury; R. Green; S. Hook; B. Oaida; W. Wu; A. Gunderson; M. Chodas, Global cloud cover for assessment of optical satellite observation opportunities: A HyspIRI case study. Remote Sens. Environ. 2012, $126,62-71$.

12. M. King, S. Platnick, W. Menzel, S. Ackerman, and P. Hubanks; M. Chodas, Spatial and temporal distribution of clouds observed by MODIS onboard the Terra and Aqua satellites. IEEE Trans. Geosci. Remote Sens. 2013, 51, 3826-3852.

13. E. Cadau; G. Laneve, Improved MSG-SEVIRI images cloud masking and evaluation of its impact on the fire detection methods. In Proc. IGARSS, Massachusetts, U.S.A., 6-11 July 2008.

14. Xiangxin $\mathrm{Xu}$; Chunqiang Yuan; Xiaohui Liang; Xukun Shen, Rendering and Modeling of Stratus Cloud Using Weather Forecast Data. In Proc. Int. Conf. on Virtual Reality and Visualization, Fujian, China, 17-18 October 2015.

15. Hongda Shen; W. David Pan; Yi Wang, A Novel Method for Lossless Compression of Arbitrarily Shaped Regions of Interest in Hyperspectral Imagery. In Proc. IEEE Southeast Con, Florida, USA, 9 - 12 April 2015. 
16. Haoyang Li; Hong Zheng; Chuanzhao Han, Adaptive run-length encoding circuit based on cascaded structure for target region data extraction of remote sensing image. In Proc. International Conference on Integrated Circuits and Microsystems, Chengdu, China, 25-28 November 2016.

17. M. Wang; W. Shi, Cloud masking for ocean color data processing in the coastal regions. IEEE Trans. Geosci. Remote Sens. 2006, 44, 3105-3196.

18. S. A. Ackerman; K. I. Strabala; W. P. Menzel; R. A. Frey; C. C. Moeller; L. E. Gumley, Discriminating clear sky from clouds with MODIS. J. Geophys. Res. Atmosp. 1998, 103, 32141-32157.

19. S. Ackerman; R. Holz; R. Frey; E. Eloranta; B. Maddux; M. McGill, Cloud detection with MODIS. Part II: Validation. J. Atmosp. Ocean. Technol. 1998, 103, 1073-1086.

20. R. A. Frey; S. A. Ackerman; Y. Liu; K. I. Strabala; H. Zhang; J. R. Key; X. Wang, Cloud detection with MODIS. Part I: Improvements in the MODIS cloud mask for collection 5. J. Atmosp. Ocean. Technol., 2008, 25, 1057-1072.

21. Jing Wei; Lin Sun; Chen Jia; Yikun Yang; Xueying Zhou; Ping Gan; Shangfeng Jia; Fangwei Liu; Ruibo Li, Dynamic threshold cloud detection algorithms for MODIS and Landsat 8 data. IEEE International Geoscience and Remote Sensing Symposium, Beijing, China, 10-15 July 2016.

22. M. K. Griffin; H. K. Burke; D. Mandl; J. Miller, Cloud cover detection algorithm for EO-1 hyperion imagery. in Proc. 17th SPIE AeroSense Conf. Algorithms Technol. Multispectral, Hyperspectral Ultraspectral Imagery IX, Orlando, FL, USA, 21-25 July 2003.

23. T. Doggett; R. Greeley; S. Chien; B. Cichy; A. Davies; G. Rabideau; R. Sherwood; D. Tran; V. Baker; J. Dohm; F. Ip, Autonomous on-board detection of cryospheric change. in Remote Sens. Environ. 2006, 101, 447-462.

24. F. Ip; J. Dohm; V. Baker; T. Doggett; A. Davies; R. Castao; S. Chien; B. Cichy; R. Greeley; R. Sherwood; D. Tran; G. Rabideau, Flood detection and monitoring with the autonomous sciencecraft experiment onboard EO-1. Remote Sens. Environ. 2006, 101, 463-481.

25. Richard R. Irish., Landsat 7 automatic cloud cover assessment. Algorithms for Multispectral, Hyperspectral, and Ultraspectral Imagery, Proceedings of SPIE, Orlando, FL USA, 24, April, 2000.

26. E. El-Araby; M. Taher; T. El-Ghazawi; J. Le Moigne, Landsat 7 automatic cloud cover assessment. Algorithms for Multispectral, Hyperspectral, and Ultraspectral Imagery, Proceedings of SPIE, Orlando, FL USA, 24, April, 2000.

27. Juan Deng, Hongchen Wang, Jun Ma, An Automatic cloud detection algorithm for Landsat Remote Sensing Image. 4th International Workshop on Earth Observation and Remote Sensing Applications, Guangdong, China, 11-14 Dec. 2016.

28. L. Gómez-Chova; G. Camps-Valls; J. Calpe-Maravilla; L. Guanter; J. Moreno, Cloud-screening algorithm for ENVISAT/MERIS multispectral images. IEEE Trans. Geosci. Remote Sens., 2007, 45, 4105-4118.

29. T. Taylor; C. O’Dell; D. O’Brien; N. Kikuchi; T. Yokota; T. Nakajima; H. Ishida; D. Crisp; T. Nakajima, Comparison of cloud-screening methods applied to GOSAT near-infrared spectra. IEEE Trans. Geosci. Remote Sens., 2012, 50, 295-309.

30. P. Minnis; Q. Z. Trepte; S. Sun-Mack; Y. Chen; D. R. Doelling; D. F. Young; D. A. Spangenberg; W. F. Miller; B. A. Wielicki; R. R. Brown; S. C. Gibson; E. B. Geier, Cloud detection in nonpolar regions for CERES using TRMM VIRS and terra and aqua MODIS data. IEEE Trans. Geosci. Remote Sens., 2008, 46, 3857-3884.

31. J. Lee; R.Weger; S. Sengupta; R.Welch, A neural network approach to cloud classification. IEEE Trans. Geosci. Remote Sens., 1990, 28, 846-855.

32. J. V. Martins; D. Tanré; L. Remer; Y. Kaufman; S. Mattoo; R. Levy, MODIS cloud screening for remote sensing of aerosols over oceans using spatial variability. Geophys. Res. Lett., 2002, 29, MOD4-1-MOD4-4.

33. Jinhu Bian; Ainong Li; Qiannan Liu; Chengquan Huang, Cloud and Snow Discrimination for CCD Images of HJ-1A/B Constellation Based on Spectral Signature and Spatio-Temporal Context. Remote Sensing, 2016, 8, 1-31.

34. F. Murtagh, D. Barreto, and J. Marcello, Decision boundaries using Bayes factors. IEEE Trans. Geosci. Remote Sens., 2003, 41, 2952-2958.

35. Haoyang Yu; Lianru Gao; Jun Li; Shansan Li; Bing Zhang, Spectral-Spatial Hyperspectral Image Classification Using Subspace-Based Support Vector Machines and Adaptive Markov Random Fields. Remote Sensing., 2016, 8, 1-21. 
36. C. Merchant, A. Harris, E. Maturi, and S. MacCallum, Probabilistic physically based cloud screening of satellite infrared imagery for operational sea surface temperature retrieval. Q. J. R. Meteorol. Soc., 2005, 131, 2735-2755.

37. David R. Thompson; Robert O. Green; Didier Keymeulen; Sarah K. Lundeen; Yasha Mouradi; Daniel Cahn Nunes; Rebecca Castaño; Steve A. Chien, Rapid Spectral Cloud Screening Onboard Aircraft and Spacecraft. IEEE Trans. on Geosci. and Remote Sens., 2014, 52, 6779 - 6792.

38. Li Wei; Wu Guodong; Zhang Fan; Qian Du, Hyperspectral Image Classification Using Deep Pixel-Pair Features. IEEE Trans. on Geosci. and Remote Sens., 2017, 55, 645-657.

39. Bin Pan; Zhenwei Shi; Xia Xu, R-VCANet: A New Deep-Learning-Based Hyperspectral Image Classification Method. IEEE Journal of Selected Topics in Applied Earth Observations and Remote Sensing, 2017, 10 , 1975 - 1986.

40. Pasquale L. Scaramuzza; Michelle A. Bouchard; John L. Dwyer, Development of the Landsat Data Continuity Mission Cloud-Cover Assessment Algorithms. IEEE Trans. on Geosci. and Remote Sens. 2012, 50 , 1140-1157.

41. Gao, X.J.; Wan, Y.C.; Zheng, X.Y, Real-Time automatic cloud detection during the process of taking aerial photographs. Spectrosc. Spectr. Anal. 2014, $34,1909-1913$.

42. Liu, J., Improvement of dynamic threshold value extraction technic in FY-2 cloud detection. J. Infrared Millim. Waves. 2010, 29 , 288-292.

43. Haoyang Yu, Lianru Gao, Jun Li, Shan Shan Li, Bing Zhang and Jón Atli Benediktsson, Spectral-Spatial Hyperspectral Image Classification Using Subspace-Based Support Vector Machines and Adaptive Markov Random Fields. Remote Sensing. 2016, 8 , 1-21.

44. Tarabalka, Y.; Benediktsson, J.A.; Chanussot, J., Spectral-spatial classification of hyperspectral imagery based on partitional clustering techniques. IEEE Trans. Geosci. Remote Sens. 2009, 47 , 2973-2987.

45. Hu Haifeng; Zhao Jianping; Wu Dongmei; Lu Na; Qin Yongyi, Implementation of Arccosine Function Based on FPGA. Electronic Technology. 2013, 6 , 5-8.

46. Tang Wen-ming, Liu Gui-xiong, FPGA Fixed-Point Technology of Exponential Function Achieved by CORDIC Algorithm. J. of South China University of Technology(Natural Science Edition), 2016, 44, 5-8.

47. Peter Malík, High throughput floating point exponential function implemented in FPGA. IEEE Computer Society Annual Symposium on VLSI, Montpellier, France, 08 - 10 Jul 2015. 